\title{
Hypoxia switches glucose depletion-induced necrosis to phosphoinositide 3-kinase/Akt-dependent apoptosis in A549 lung adenocarcinoma cells
}

\author{
CHO HEE KIM ${ }^{1 *}$, A RA KO ${ }^{1 *}$, SU YEON LEE ${ }^{1}$, HYUN MIN JEON $^{1}$, SUN MI KIM ${ }^{1}$, \\ HYE GYEONG PARK ${ }^{2}$, SONG IY HAN ${ }^{3}$ and HO SUNG KANG ${ }^{1}$ \\ ${ }^{1}$ Department of Molecular Biology, College of Natural Sciences, and Research Institute of Genetic Engineering, \\ ${ }^{2}$ Nanobiotechnology Center, Pusan National University, Pusan 609-735; ${ }^{3}$ Research Center \\ for Resistant Cells, Chosun University, Gwangju 501-759, Korea
}

Received August 27, 2009; Accepted October 13, 2009

DOI: 10.3892/ijo_00000482

\begin{abstract}
In solid tumours, necrosis is commonly found in the core region in response to metabolic stress that results from oxygen and glucose depletion (OGD) due to insufficient vascularization and has been implicated in tumour progression. We have previously shown that metabolic stress due to glucose depletion (GD) induces necrosis and HMGB1 release through mitochondrial ROS production in A549 lung adenocarcinoma cells. In this study, we examined the effects of hypoxia on GD-induced necrosis and show that hypoxia prevented GD-induced mitochondrial ROS production, HMGB1 release, and necrosis and switched the cell death mode to apoptosis that is dependent on caspase-3 and -9 . We further found that inhibition of ERK1/2 by U0126 abolished the effects of hypoxia to switch the cell death mode and to suppress mitochondrial ROS production, indicating an important role(s) of the ERK pathway in cell death mode determination. We also found that during OGD-induced apoptosis the prosurvival protein kinase Akt is activated and inhibition of Akt by the phosphoinositide 3-kinase (PI3K) inhibitors LY294002 and wortmannin prevent OGD-induced apoptosis, caspase-3 and -9 activation, and nuclear translocation of AIF and EndoG. Similar inhibitory effects of PI3K inhibitors were observed in A549 cells that underwent apoptosis when treated with GD in the presence of NAC (a general antioxidant) or catalase $\left(\mathrm{a} \mathrm{H}_{2} \mathrm{O}_{2}\right.$ scavenger), or in the presence of active PKC by treatment with phorbol-12-myristate-13-acetate, indicating
\end{abstract}

Correspondence to: Dr Ho Sung Kang, Department of Molecular Biology, College of Natural Sciences, Pusan National University, Pusan 609-735, Korea

E-mail: hspkang@pusan.ac.kr

${ }^{*}$ Contributed equally

Key words: hypoxia, glucose depletion, necrosis, apoptosis, high mobility group box 1 , PI3K a crucial role(s) of the PI3K-Akt pathway in OGD-indcued apoptosis. In conclusion, our results demonstrate that hypoxia switches GD-induced necrosis to apoptosis and ERK1/2 and PI3K-Akt exert anti-necrotic and pro-apoptotic activities in the cell death, respectively.

\section{Introduction}

Cell death occurs via two distinct pathways, apoptosis or necrosis. Apoptosis is a well-defined cell death program that is characterized by chromatin condensation/fragmentation and formation of apoptotic bodies that are removed by phagocytic cells (i.e., macrophages) before plasma membrane integrity is lost and therefore in most cases does not cause inflammation $(1,2)$. Apoptotic cell death includes a proteolytic system consisting of caspases that is activated via either an extrinsic pathway, which depends on cell surface death receptors and caspase- 8 activation or an intrinsic mitochondrial pathway, which depends on caspase-9 $(1,2)$. Caspase-3, a downstream effector of caspase- 8 and -9 , is a main executioner that cleaves many cellular proteins including poly(ADP-ribose) polymerase (PARP) and $\beta$-catenin $(1,2)$. In addition, two major apoptotic nucleases, caspase-activated DNase (CAD) and endonuclease $\mathrm{G}$ (EndoG), are responsible for DNA fragmentation $(3,4)$. In normal cells, CAD exists in the nucleus as a heterodimer with an inhibitor of CAD (ICAD), and when ICAD is cleaved by active caspase- 3 in response to apoptotic stimuli, CAD is activated. In contrast, EndoG in the mitochondrial inter-membrane space is translocated to the nucleus upon apoptotic stresses with another apoptotic molecule, apoptosis-inducing factor (AIF) $(3,4)$. Apoptosis is regulated by a number of factors including the tumour suppressor p53 $(1,2)$ and Bcl-2 family proteins including anti-apoptotic proteins, such as Bcl-2, and pro-apoptotic BAX and BAK (5). In addition, the phosphoinositide 3-kinase (PI3K) pathway involving Akt/PKB kinase antagonizes apoptotic cell death in response to many different toxic stresses (6-8). Unlike apoptosis, during necrosis, the cell membrane is ruptured, and the cytoplasmic contents [e.g., a nuclear protein high mobility group box 1 (HMGB1)] are released into the extracellular space causing a massive inflammatory response (9-12). 
Recently, necrosis and inflammation has been suggested to promote tumour growth and angiogenesis through the tumour promoting activity of HMGB1 and by increasing probability of proto-oncogenic mutation (13-18). Indeed, increased expression of HMGB1, as well as its receptor RAGE (receptor for advanced glycation end-products), has been observed in many different tumours including hepatomas and prostate cancer, which correlates with invasiveness and poor outcome when RAGE is expressed in conjunction with its ligand $(19,20)$. Since the consequences of necrotic and apoptotic cell death are quite different for a whole organism, it is crucial to decide whether cells die by necrosis or apoptosis particularly with respect to the development of tumour in an organism.

In solid tumours, necrosis is commonly found in the core region in response to metabolic stress that results from oxygen and glucose depletion (OGD) due to insufficient vascularization (21-25). We have previously demonstrated that GD induced necrosis through mitochondrial ROS production in A549 lung carcinoma cells and that NAC (a general antioxidant) or catalase (a $\mathrm{H}_{2} \mathrm{O}_{2}$ scavenger) could switch GDinduced necrosis to apoptosis (26). We also showed that protein kinase C-dependent ERK $1 / 2$ activation switched GD-induced necrosis to apoptosis through inhibiting ROS production, possibly by inducing MnSOD expression and preventing GD-induced down-regulation of CuZnSOD, demonstrating a critical role(s) of ROS in the determination of GD-induced cell death mode (26). Here we examined the effects of hypoxia on GD-induced necrosis and show that hypoxia prevented GD-induced necrosis and HMGB1 release. We further show that OGD-induced apoptosis is regulated by the PI3K-Akt/PKB signal pathway.

\section{Materials and methods}

Cell culture and drug treatment. Human lung adenocarcinoma A549 cells were obtained from American Type Culture Collection and maintained in RPMI-1640 containing 10\% FBS as described previously $(26,27)$. For GD treatment, cells were rinsed twice with glucose-free medium, and incubated in GD medium (glucose-free RPMI-1640 or DMEM containing $10 \%$ dialyzed and heat-inactivated FBS and 1\% PS). For OGD, cells were exposed to OGD using an anaerobic chamber (Forma 1025) containing 2\% $\mathrm{O}_{2}, 5 \% \mathrm{CO}_{2}$ and $93 \% \mathrm{~N}_{2}$ in $37^{\circ} \mathrm{C}$ for the indicated times. A549 cells were pretreated with inhibitors including LY294002 (Sigma, $20 \mu \mathrm{M}$ ), wortmannin (Sigma, $0.2 \mu \mathrm{M}$ ), U0126 (Sigma, $20 \mu \mathrm{M}$ ), z-VAD-fmk (Calbiochem, $20 \mu \mathrm{M}$ ), z-DEVD-fmk (Calbiochem, $20 \mu \mathrm{M}$ ), z-LEHD-fmk (Calbiochem, $50 \mu \mathrm{M}$ ), N-acetyl-L-cysteine (Sigma, $10 \mathrm{mM}$ ), catalase (Sigma, 1,000 $\mu \mathrm{g} / \mathrm{ml}$ ) for $1 \mathrm{~h}$, and then treated with GD or OGD in the presence of the inhibitors. For activation of PKC, cells were pretreated with phorbol12-myristate-13-acetate (PMA, Calbiochem, $100 \mathrm{nM}$ ) for 30 min and washed with glucose-free RPMI-1640 twice and incubated in GD medium.

SDS-PAGE and Western blot analysis. Western blotting was performed with antibodies to HMGB1 (BD Pharmingen, CA), CuZnSOD, MnSOD, catalase (Santa Cruz, CA), ERK1/2, phosphoERK1/2 (Cell Signaling, MA), Akt, phopsho-Akt
(Cell Signaling, MA), PARP (Santa Cruz), active caspase-3, active caspase-9 (Cell Signaling), and B-tubulin (Sigma). After washing 3 times in Tris-buffered saline (TBS) with $0.1 \%$ Tween-20, primary antibody was detected using 1:1,000 diluted HRP-conjugated secondary antibodies and visualized with the enhanced chemiluminescence detection system (Amersham-Pharmacia Biotech, Buckinghamshire, UK).

HMGB1 and CUZnSOD release, intracellular ROS and mitochondrial ROS production, Hoechst 33342 (HO) and propidium iodide (PI) staining, and AIF and EndoG translocation assay. HMGB 1 and CuZnSOD release assay and HO/PI stainging was performed as described previously $(26,27)$. For immunofluorescence and confocal microscopy, A549 cells grown for $24 \mathrm{~h}$ on coverslips were incubated in GD medium for the indicated times. The cells were fixed with $3.7 \%$ formaldehyde and permeabilized with $0.2 \%$ Triton $\mathrm{X}-100$ for $30 \mathrm{~min}$ on ice. After blocking with $2 \%$ bovine serum albumin in $0.1 \%$ PBST, the cells were incubated with the primary antibodies against $\mathrm{AIF}$ and EndoG overnight at $4^{\circ} \mathrm{C}$, then washed 3 times in PBST and incubated with the secondary antibodies conjugated with fluorescein isothiocyanate (FITC; Sigma) for $1 \mathrm{~h}$. Nuclei were then stained with $2.5 \mathrm{mg} / \mathrm{ml}$ PI for $10 \mathrm{~min}$. After washing 3 times with PBS, coverslips were mounted onto microscopic slides using Cristal mounting reagent (Biomeda, Foster City, CA). The slides were observed by a fluorescence microscope (Carl Zeiss) and photographed by AxioCam (Carl Zeiss). Intracellular $\mathrm{H}_{2} \mathrm{O}_{2}, \mathrm{O}_{2}{ }^{-}$and mitochondrial ROS were detected using the 2',7'-dichlorofluorescin diacetate (DCFH-DA) (Molecular Probes, $50 \mu \mathrm{M}$ ), dihydroethidium (HE, Molecular Probes, $10 \mu \mathrm{M}$ ), or MitoTracker Red CM-H2XRos (Molecular Probe, $50 \mathrm{nM}$ ) under a fluorescence microscope.

\section{Results and Discussion}

Hypoxia switches glucose deprivation-induced necrosis to apoptosis in A549 cells. GD induced cell death mode (apoptotic or necrotic) in A549 cell was determined by Hoechst33342 (HO)/propidium iodidie (PI) double staining. HO crosses the plasma membrane of all cells regardless of cell damage, but, polar PI only penetrates cells with damaged membranes. Thus, intact blue nuclei, condensed/fragmented blue nuclei, condensed/fragmented pink nuclei, and intact pink nuclei were considered viable, early apoptotic, late apoptotic, and necrotic cells, respectively. In the presence of glucose, most of cells had intact blue nuclei, whereas cells cultured with GD medium exhibited intact pink nuclei, indicating that GD induces necrosis in A549 cells (Fig. 1a and b). We examined the effects of hypoxia on GD-induced necrosis. We found that hypoxia prevents GD-induced cell rounding and caused blebbing of the plasma membrane after $15 \mathrm{~h}$ OGD treatment (data not shown). Hypoxia markedly prevented GD-induced necrosis and switched the cell death mode to apoptosis, as observed by HO/PI staining (Fig. 1a and b). Whereas necrosis could be observed after $12 \mathrm{~h}$ GD treatment, apoptosis was observed to start at $15 \mathrm{~h}$ after OGD treatment, indicating that necrosis and apoptosis are initiated at different time-points possibly through different mechanisms. Necrosis-to-apoptosis switch was confirmed by HMGB1 release assay. HMGB1 is 


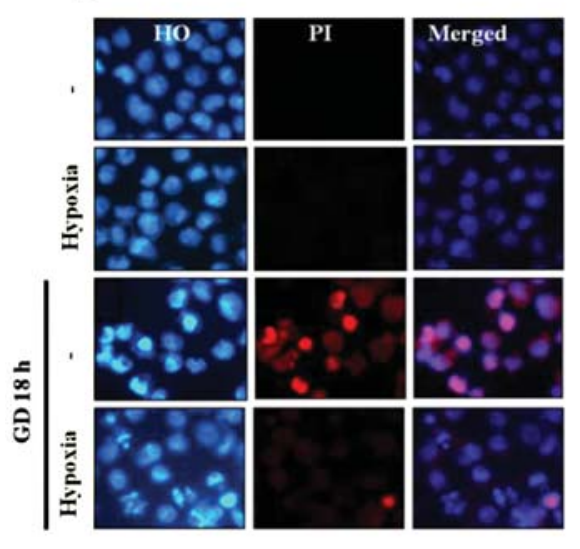

b

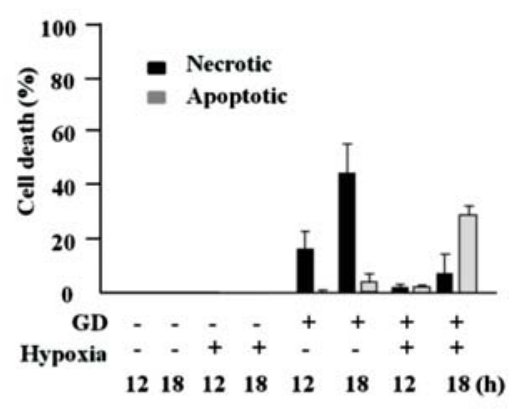

c

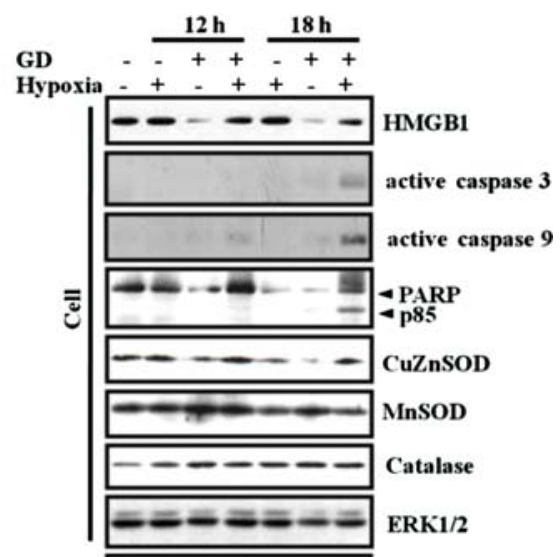

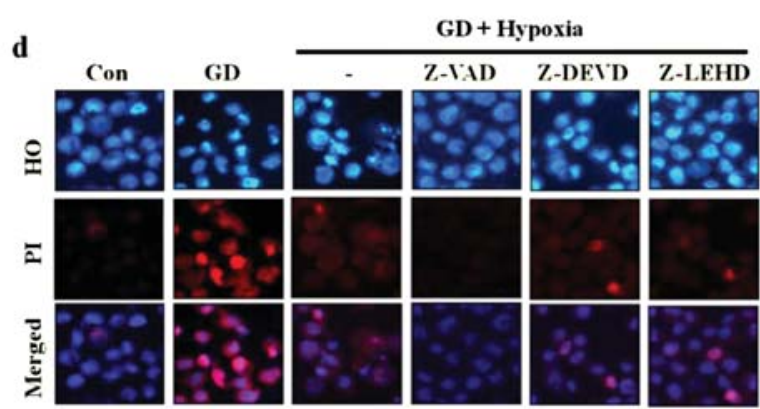

f
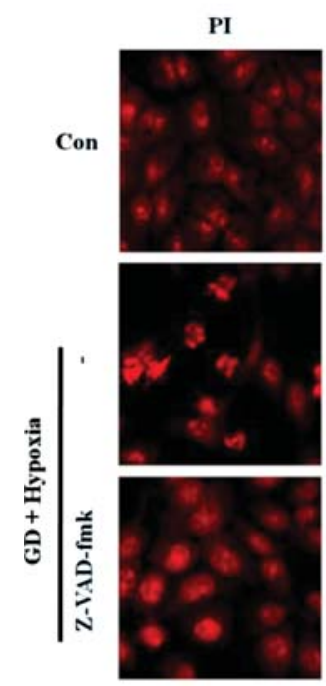

AIF
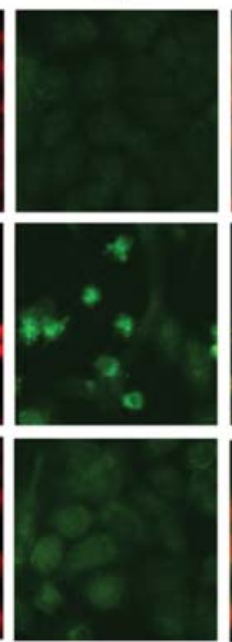

Merged
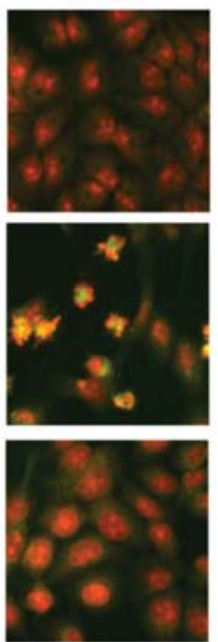

e

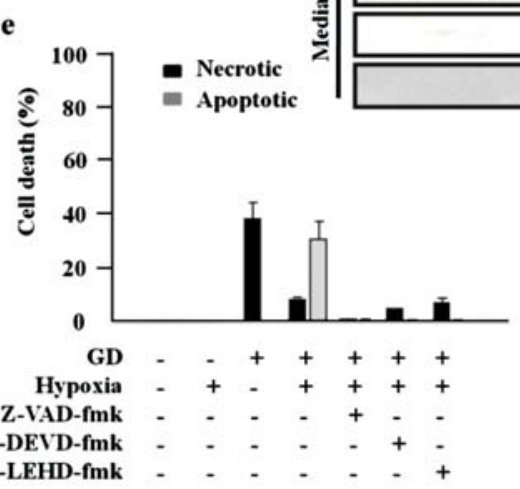

HMGB1

CuZnsoD ERK1/2

Figure 1. Hypoxia switches GD-induced necrosis to apoptosis. (a and b) A549 cells were exposed to GD or OGD for the indicated times and stained with HO/PI, and apoptotic and necrotic cells were scored under a fluorescence microscope. Results (500-800 cells in each group) are expressed as the means \pm SEM from three independent experiments. (c) A549 cells were exposed to GD or OGD for the indicated time and both the cells and medium bathing the cells were prepared as described in Materials and methods and analyzed by Western blotting using antibodies to HMGB1, caspase-3, caspase-9, PARP, CuZnSOD, MnSOD, catalase, and ERK1/2. (d and e) A549 cells were pretreated with z-VAD-fmk, z-DEVD-fmk, or z-LEHD-fmk and exposed to OGD for 15 h. The cells were stained with HO/PI and observed under a fluorescence microscope and apoptotic and necrotic cells were scored. Results (500-800 cells in each group) are expressed as the means \pm SEM from three independent experiments. (f) A549 cells were exposed to OGD for 18 h and fixed, and stained with AIF/PI or EndoG/PI as described in Materials and methods. The cells were observed under a fluorescence microscope.

a nuclear protein that plays a role(s) in DNA bending and transcription, but is leaked rapidly into the medium when membrane integrity is lost in permeabilized or necrotic cells. Hypoxia prevented HMGB1 release into the extracellular space and reduction its cellular levels in response to GD (Fig. 1c). Previously, we have shown that CuZnSOD, but not other anti- oxidants MnSOD and catalase, is released into the extracellular space in an active form upon GD, thereby accelerating ROS (possibly $\mathrm{O}_{2}^{-}$) damage and facilitating necrotic cell death [(26) and Fig. 1c]. Hypoxia suppressed GD-induced CuZnSOD release into the extracellular space of cells (Fig. 1c). To further confirm hypoxia-mediated necrosis to apoptosis switch, nuclear 


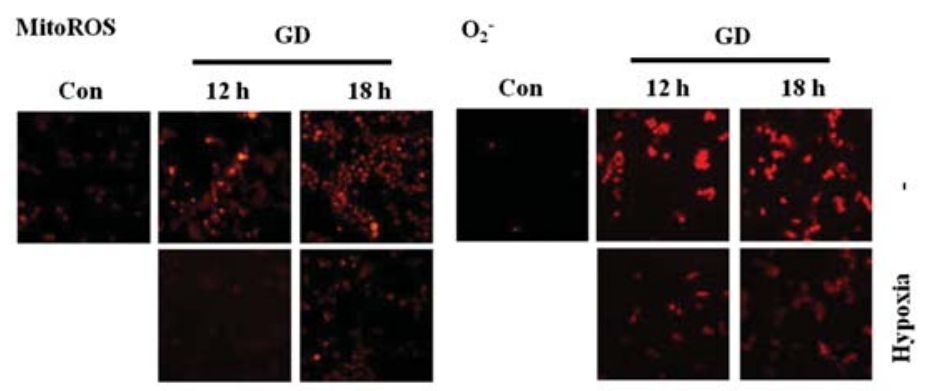

b
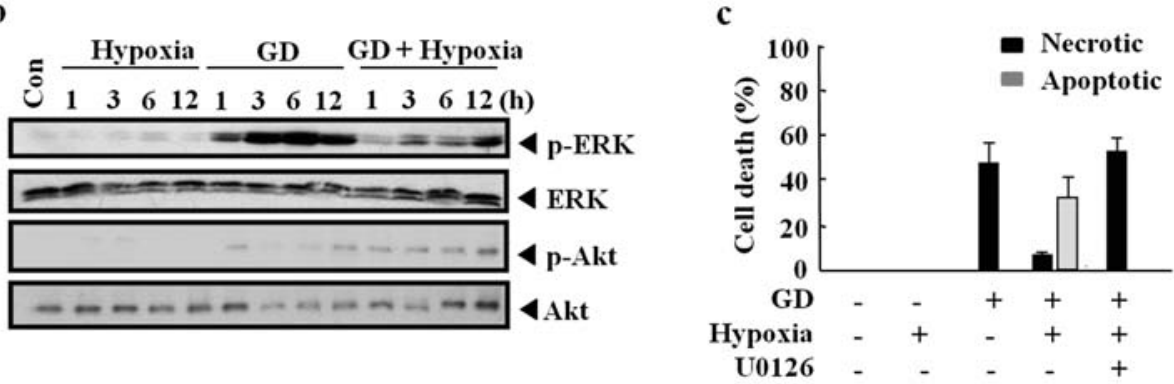

d

e
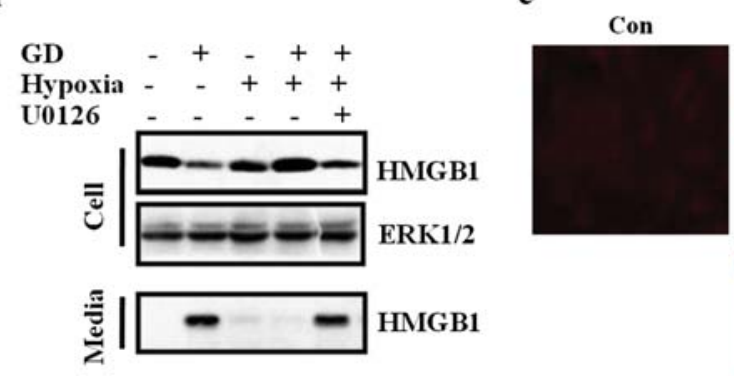

GD

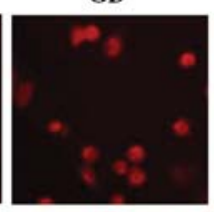

GD + Hypoxia GD + Hypoxia + U0126
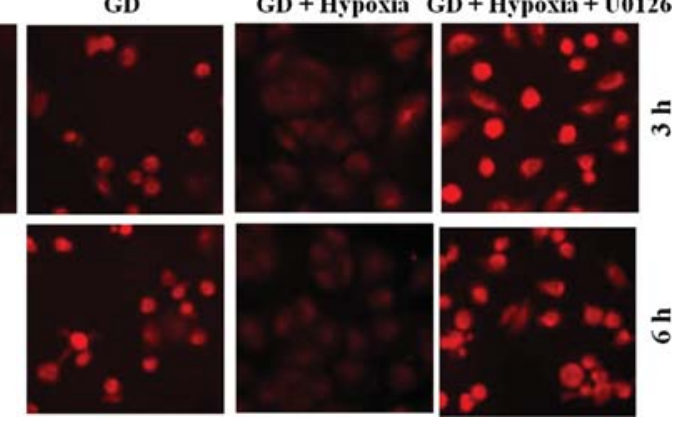

Figure 2. (a) A549 cells were exposed GD or OGD for the indicated times and treated with MitoTracker Red CM- $\mathrm{H}_{2} \mathrm{XRos}$ or dihydroethidium (HE) and observed under a confocal microscope to detect mitochondrial ROS (Mito ROS) and intracellular $\mathrm{O}_{2}^{-}$, respectively. (b) A549 cells were exposed to hypoxia, GD, or OGD for the indicated times and the resulting cell lysates were analyzed by SDS-PAGE and Western blotting with antibodies to p-ERK1/2, ERK1/2, p-Akt/PKB, and Akt/PKB. (c) A549 cells were pretreated with U0126 for $1 \mathrm{~h}$ and exposed GD or OGD for $18 \mathrm{~h}$ and then stained with HO/PI and observed with fluorescence microscopy and apoptotic and necrotic cells were scored. Results (500-800 cells in each group) are expressed as the means \pm SEM from three independent experiments. (d) A549 cells were exposed to GD or OGD for the indicated times and both the cells and medium bathing the cells were prepared as described in Materials and methods and analyzed by Western blotting using antibodies to HMGB1 and ERK1/2. (e) A549 cells were pretreated with U0126 for $1 \mathrm{~h}$ and exposed GD or OGD for $18 \mathrm{~h}$ and treated with MitoTracker Red CM-H2XROS and observed under a confocal microscope to detect mitochondrial ROS (Mito ROS).

translocation of AIF and EndoG, two major apoptotic nucleases that reside in the mitochondrial inter-membrane space in normal cells, was performed in A549 cells. As shown in Fig. 1f, OGD induced nuclear translocation of EndoG and AIF. Then, we examined whether GD-induced apoptosis is linked to either an extrinsic pathway, which activates caspase-8 $(1,2)$ or an intrinsic mitochondrial pathway, which activates caspase-9 $(1,2)$. OGD caused activation of caspase-9 and caspase-3 (Fig. 1c), but not caspase-8 (data not shown). Furthermore, p85 cleavage product of PARP was also detected in cells exposed to OGD (Fig. 1c). In addition, a broad caspase inhibitor (z-VAD-fmk), caspase-3 inhibitor (z-DEVD-fmk), and caspase-9 inhibitor (z-LEHD-fmk) completely suppressed OGD-induced apoptosis (Fig. 1d and e), supporting that caspase-3 and -9 play a critical role in OGD-induced apoptosis. OGD-induced nuclear translocation of EndoG and AIF was also prevented by a broad caspase (zVAD-fmk) (Fig. 1f), but to a lesser extent, by caspase-3 inhibitor (z-DEVD-fmk) and caspase-9 inhibitor (z-LEHD-fmk) (data not shown).

Hypoxia inhibits GD-induced mitochondrial ROS production. Previous studies showed that GD induced production of mitochondrial ROS $(28,29)$ and could be switched to apoptosis when ROS production was abolished by treatment with antioxidants including NAC and catalase, indicating a critical role(s) of ROS in the determination of GD-induced cell death mode (26). Thus, we investigated whether the effects of hypoxia is linked to regulation of GD-induced ROS production and found that hypoxia inhibits GD-induced production of mitochondrial ROS and intracellular $\mathrm{O}_{2}^{-}$, as determined using MitoTracker Red CM-H2XRos or dihydroethidium (HE) (Fig. 2a). 

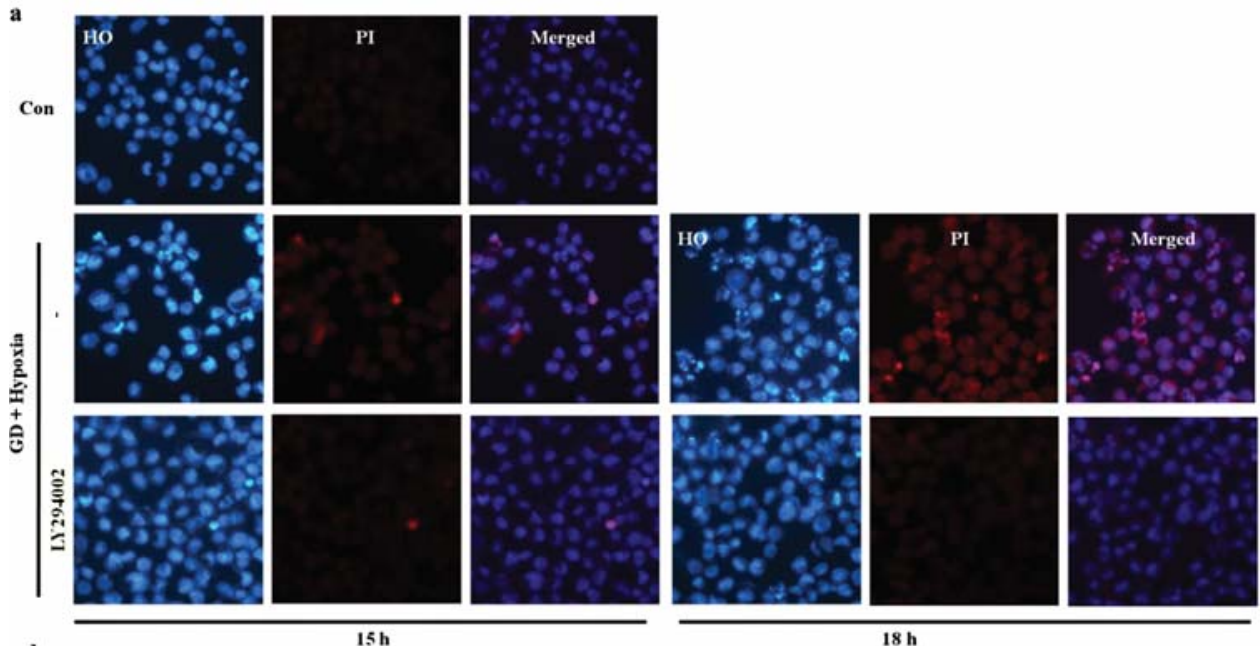

$18 \mathrm{~h}$
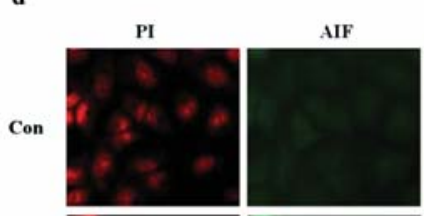

Merged
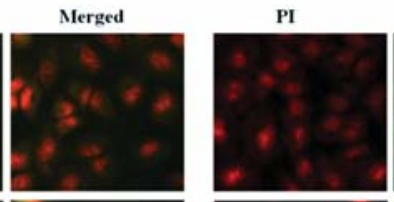

EndoG

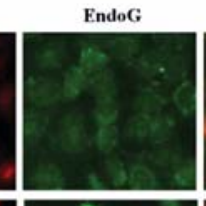

Merged
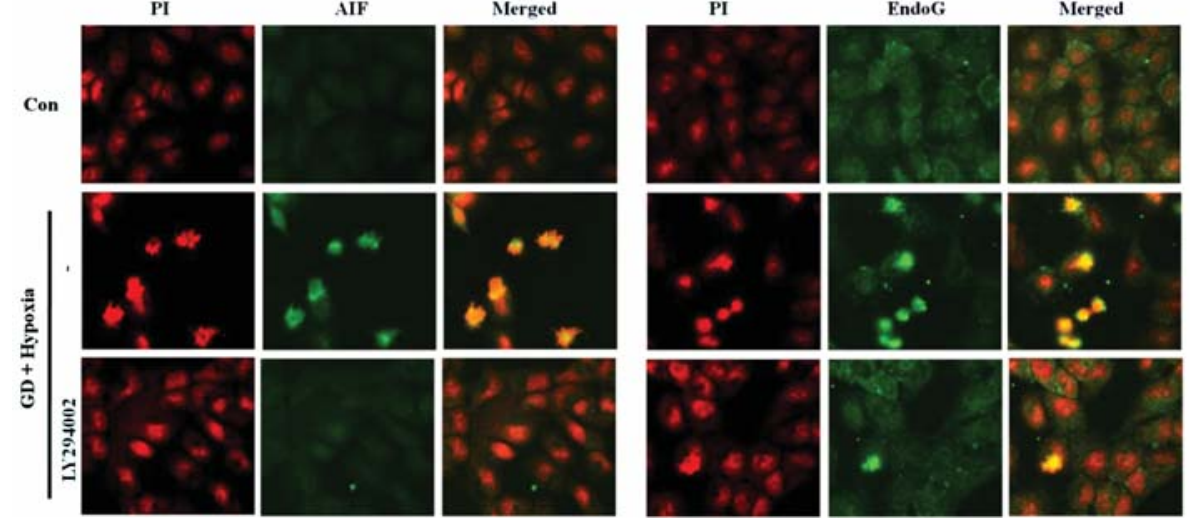

Figure 3. (a and b) A549 cells pretreated with LY294002 or wortmannin for $1 \mathrm{~h}$ were exposed OGD for $18 \mathrm{~h}$ and then stained with HO/PI and observed with fluorescence microscopy and apoptotic and necrotic cells were scored. Results (500-800 cells in each group) are expressed as the means \pm SEM from three independent experiments. (c) A549 cells were exposed to GD or OGD for indicated time and the resulting cell lysates analyzed by Western blotting using antibodies to caspase-3, caspase-9, PARP, and tubulin. (d) A549 cells pretreated with LY294002 for $1 \mathrm{~h}$ were exposed OGD for $15 \mathrm{~h}$. The cells were fixed, and stained with AIF/PI or EndoG/PI as described in Materials and methods. The cells were observed under a fluorescence microscope.

ERK1/2 plays an important role(s) in GD-induced cell death mode determination. Previous studies have shown that the MEK1/2-extracellular signal-regulated kinase 1/2 (ERK1/2) pathway exert an inhibitory effect on GD-induced necrosis $(26,30)$. Thus, we investigated the role(s) of the MEK1/2ERK1/2 pathway in OGD-induced cell death. As shown in Fig 2b, ERK1/2 was activated through phosphorylation of Thr 202/Tyr 204 by either GD or OGD, although its activation was higher in GD-treated cells than in OGD-treated cells. We examined the effects of the MEK inhibitor U0126 on OGD-induced apoptosis. We found that U0126 abolished the effects of hypoxia to switch the cell death mode (Fig. 2c) and to suppress GD-induced HMGB1 release (Fig. 2d) and reversed the cell death mode to necrosis (Fig. 2c). Thus, ERK1/2 may act as an anti-necrotic pathway in hypoxia-mediated cell death mode switch. In addition, U0126 suppressed the activities of hypoxia to prevent mitochondrial ROS production (Fig. 2e). Thus, ERK1/2 appeared to prevent GD-induced necrosis through mitochondrial RPS production.

The PI3K-Akt pathway is activated by hypoxia and regulates $O G D$-induced apoptosis. In the course of this study, we found that Akt/PKB is activated through phosphorylation of Ser 473 by OGD (Fig. 2b). Akt/PKB is mostly activated by the phosphoinositide 3-kinase (PI3K) signaling cascade and plays a critical role(s) in cell survival and antagonizes apoptotic cell death in response to a variety of toxic stresses (6-8). Akt/PKB could be activated in response to some proapoptotic stimuli such as UV irradiation and DNA damage, and confers cancer cells with resistance to chemotherapy and interferes with intended action of pro-apoptotic stimuli (6-8). The PI3K-Akt/PKB pathway possesses anti-apoptotic activities through phosphorylating and inactivating proapoptotic molecules including BAD and GSK-3ß. Thus, we hypothesized that inhibition of the PI3K-Akt/PKB signal pathway makes cells susceptible to OGD-induced apoptosis. We examined the effects of the PI3K inhibitors LY294002 and wortmannin on OGD-induced apoptosis. However, unexpectedly, the PI3K inhibitors appeared to prevent OGDinduced apoptosis (Fig. 3a and b), caspase-3 and -9 activation and PARP cleavage (Fig. 3c), and OGD-induced nuclear translocation of EndoG and AIF (Fig. 3d), without reversing the cell death mode to necrosis (Fig. 3a), raising the possibility that the PI3K-Akt signaling may exert a pro-apoptotic activity in this type of cell death.

To further confirm the unusual pro-apoptotic activity of the PI3K-Akt signaling, we examined the effects of the PI3K inhibitors LY294002 and wortmannin on apoptosis 


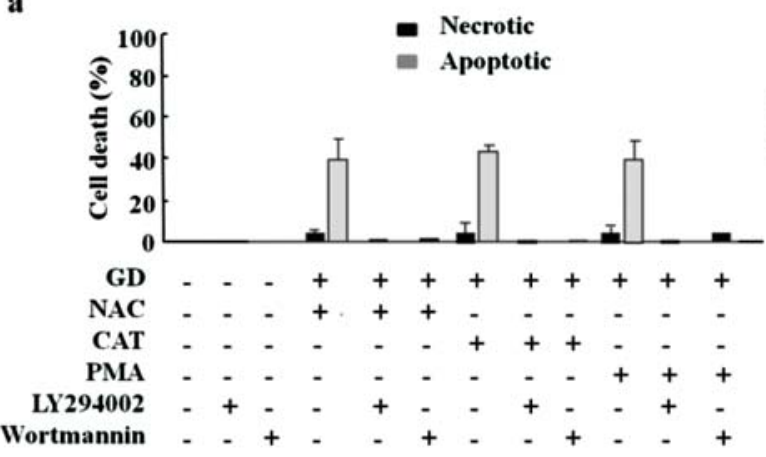

GD $\quad-1218-1218-1218(\mathrm{~h})$

CAT $\quad-1218-12+++++$

LY294002 - . - . - + + +

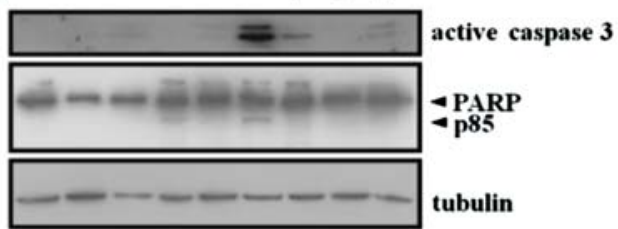

c
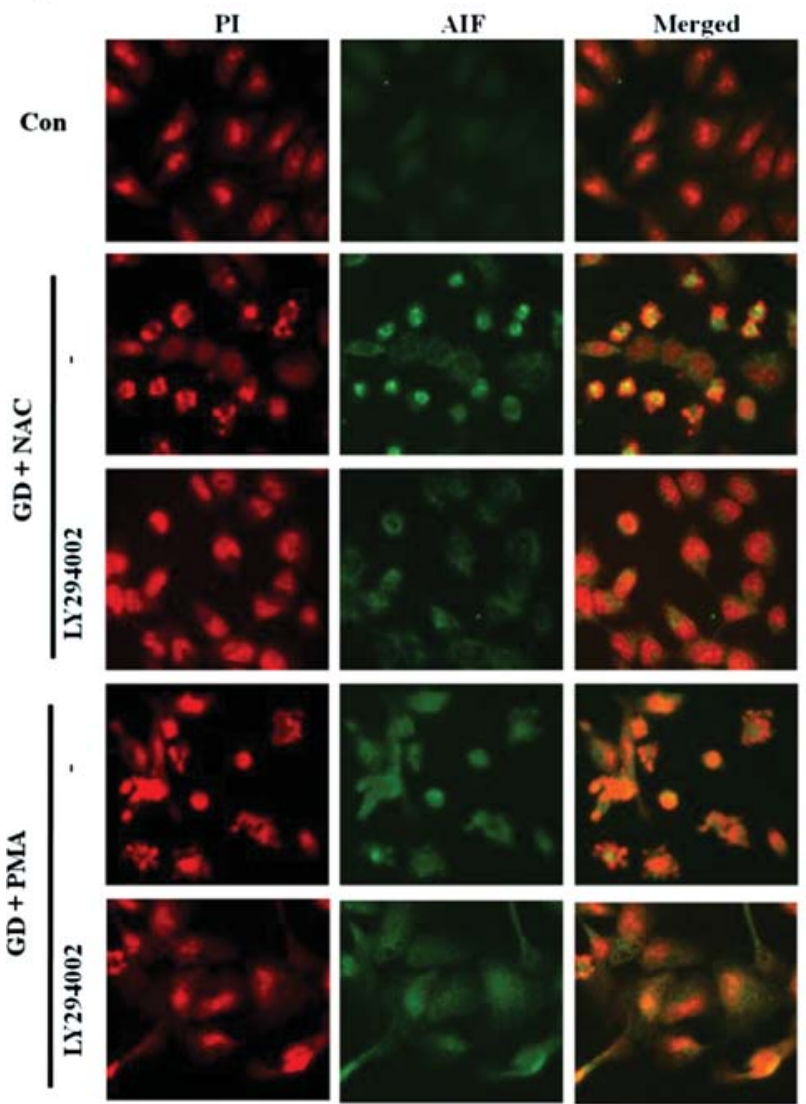

b
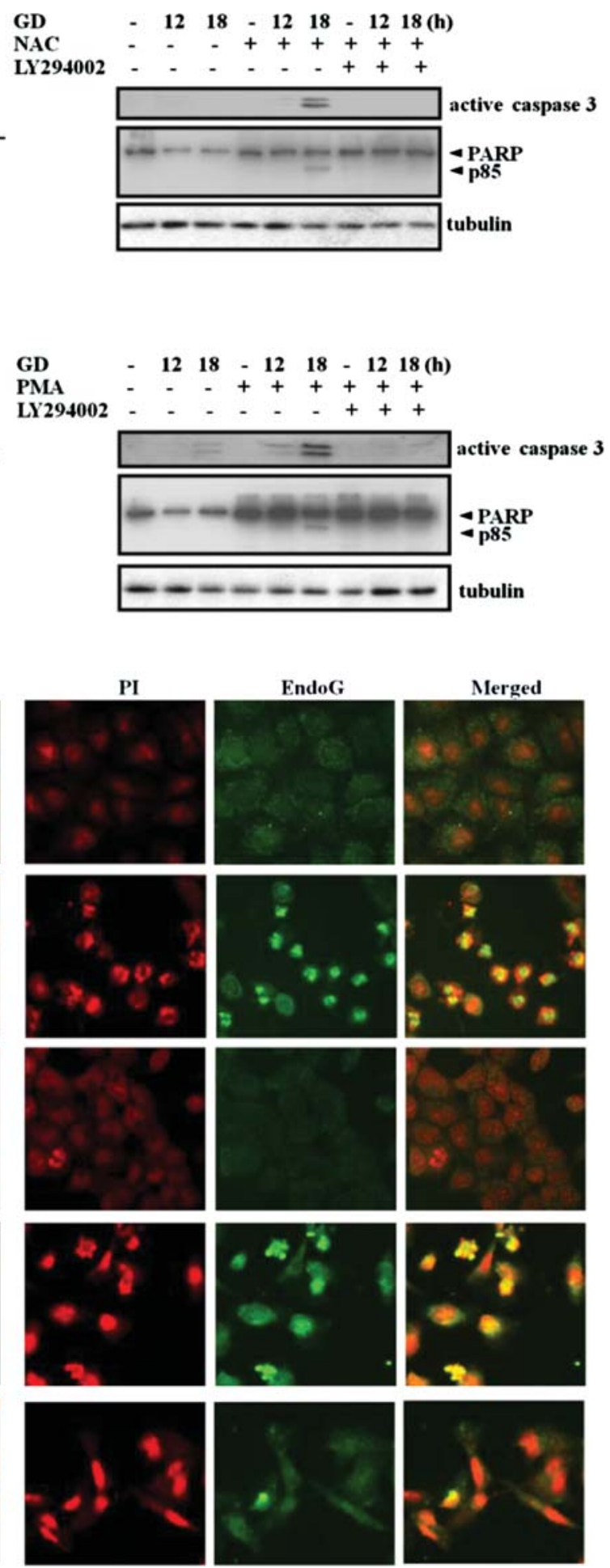

Figure 4. (a) A549 cells pretreated with LY294002 or wortmannin for $1 \mathrm{~h}$ and pretreated with NAC, catalase (CAT), or PMA were exposed OGD for $18 \mathrm{~h}$ and then stained with HO/PI and observed with fluorescence microscopy and apoptotic and necrotic cells were scored. Results (500-800 cells in each group) are expressed as the means \pm SEM from three independent experiments. (b) A549 cells pretreated with LY294002 or wortmannin for $1 \mathrm{~h}$ and pretreated with NAC, catalase (CAT) or PMA were exposed OGD for $18 \mathrm{~h}$ and then analyzed by Western blotting using antibodies to caspase-3, PARP, and tubulin. (C) A549 cells pretreated with LY294002 or wortmannin for $1 \mathrm{~h}$ and pretreated with NAC or PMA were exposed GD for $18 \mathrm{~h}$. The cells were fixed, and stained with AIF/PI or EndoG/PI as described in Materials and methods. The cells were observed under a fluorescence microscope.

that occurs when treated with GD in the presence of NAC (a general antioxidant) or catalase $\left(\mathrm{a} \mathrm{H}_{2} \mathrm{O}_{2}\right.$ scavenger), or in the presence of active PKC by treatment with phorbol-12- myristate-13-acetate. We found that the PI3K inhibitors prevented apoptosis that occurred in these conditions (Fig. 4a). The PI3K inhibitors also prevented caspase-3 activation and 


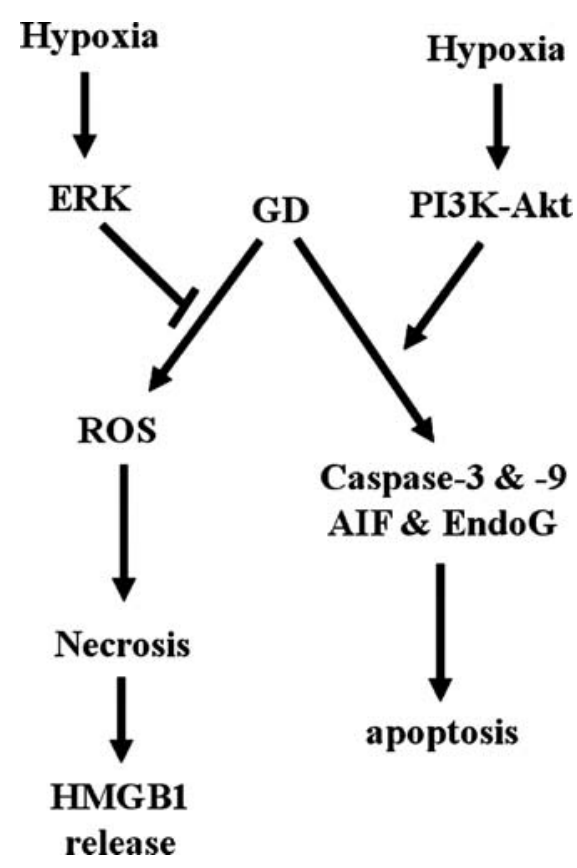

Figure 5. A schematic model for hypoxia-mediated cell death mode switch.

PARP cleavage (Fig. 4b) and nuclear translocation of EndoG and AIF (Fig. 4c). In addition, PI3K inhibition results in the prevention of GD-induced apoptosis in A549 cells that were cultured at a high density and exposed to GD (data not shown). These results demonstrated that the PI3K-Akt signal cascade exert pro-apoptotic activities. Although PI3K-Akt pathway is a typical pro-survival pathway, recent studies demonstrated that PI3K pathway is also involved in apoptotic cell death (31-33). For instance, high glucose triggers the caspase-3 activation through PI3K/Akt-mediated NF- $\kappa \mathrm{B}$ up-regulation of COX-2, which in turn facilitates human umbilical vein endothelial cells (HUVECs) apoptosis and may cause ROS generation in HUVECs through a PI3K/Akt-dependent pathway (31). In addition, inhibition of PI3K or mTOR dramatically reduced hypoxia-induced necrotic cell death in Lewis lung carcinoma cells under hypoxic conditions (32). $\mathrm{PI} 3 \mathrm{~K} / \mathrm{mTOR}$ pathway is necessary for the ability of interferon- $\alpha$ to induce a caspase-dependent apoptosis that is associated with activation of the pro-apoptotic Bak and Bax, loss of mitochondrial membrane potential, and release of cytochrome c (33). Thus, the PI3K-Akt signaling may exert a pro-apoptotic activity in apoptotic cell death that occurs due to altered cellular oxygen or glucose levels or in response to a specific set of apoptotic stimuli, although its exact molecular mechanism remains to be clarified. In conclusion, our results demonstrate that hypoxia switches GD-induced necrosis to apoptosis and ERK1/2 and PI3K-Akt exert anti-necrotic and pro-apoptotic activities in the cell death, respectively (Fig. 5).

Biological relevance of hypoxia-mediated cell death mode switch. When a solid tumour continues to grow, its center becomes distant from surrounding blood vessels thereby experiencing OGD-drived metabolic stress. Under such stressful circumstances, tumour cells adapt to survive against the potentially lethal effects of metabolic constraint or die by necrosis. In fact, necrosis is commonly found in the core region of solid tumours. Necrosis has been implicated in tumour progression and aggressiveness. Necrotic cells may attract macrophages into the tumour and necrotic cells and activated macrophages could release HMGB1 outside the cells. HMGB1 acts as a potent mediator of inflammation through binding with high affinity to RAGE, thereby potentiating angiogenesis, neoplastic growth and promoting tumour initiation, promotion, and progression. Our results showed that hypoxia could switch GD-induced necrosis to apoptosis (Fig. 1). Why does hypoxia inhibit GD-induced necrosis and switch the cell death mode to apoptosis? The reason for this phenomenon is unclear. However, since excessive inflammation and subsequent high level infiltration of monocytes and/or neutrophil can lead to tumour cytotoxicity and regression, hypoxia-mediated necrosis to apoptosis switch may suppress the development of excessive inflammation that could prevent tumour progression. In most tumours, apoptosis is commonly observed in the surroundings of the necrotic core. Thus, the ability of hypoxia to suppress metabolic stress-induced necrosis and to switch the cell death mode to apoptosis may contribute to promotion of malignant and aggressive tumour progression.

\section{Acknowledgements}

This work was supported by the Korea Research Foundation Grant funded by the Korean Government (MOEHRD, Basic Research Promotion Fund) (KRF-2007-521-C00201), and a grant from the National R\&D Program for Cancer Control, Ministry of Health and Welfare, Republic of Korea (0620010).

\section{References}

1. Hengartner MO: The biochemistry of apoptosis. Nature 407: 770-776, 2000

2. Danial NN and Korsmeyer SJ: Cell death: critical control points. Cell 116: 205-219, 2004.

3. Widlak P and Garrard WT: Discovery, regulation, and action of the major apoptotic nucleases DFF40/CAD and endonuclease G. J Cell Biochem 94: 1078-1087, 2005.

4. Arnoult D, Gaume B, Karbowski M, Sharpe JC, Cecconi F and Youle RJ: Mitochondrial release of AIF and EndoG requires caspase activation downstream of Bax/Bak-mediated permeabilization. EMBO J 22: 4385-4399, 2003.

5. Tsujimoto Y: Cell death regulation by the Bcl-2 protein family in the mitochondria. J Cell Physiol 195: 158-167, 2003.

6. Crowell JA and Steele VE: AKT and the phosphatidylinositol 3-kinase/AKT pathway: important molecular targets for lung cancer prevention and treatment. J Natl Cancer Inst 95: 252-253, 2003.

7. Clark AS, West K, Streicher S and Dennis PA: Constitutive and inducible Akt activity promotes resistance to chemotherapy, trastuzumab, or tamoxifen in breast cancer cells. Mol Cancer Ther 1: 707-717, 2002.

8. Yamaguchi $\mathrm{H}$ and Wang HG: The protein kinase PKB/Akt regulates cell survival and apoptosis by inhibiting Bax conformational change. Oncogene 20: 7779-7786, 2001.

9. Syntichaki P and Tavernarakis N: Death by necrosis. Uncontrollable catastrophe, or is there order behind the chaos? EMBO Rep 3: 604-609, 2002.

10. Kanduc D, Mittelman A, Serpico R, et al: Cell death: apoptosis versus necrosis. Int J Oncol 21: 165-170, 2002.

11. Zong WX and Thompson CB: Necrotic death as a cell fate. Genes Dev 20: 1-15, 2006.

12. Golstein P and Kroemer G: Cell death by necrosis: towards a molecular definition. Trends Biochem Sci 32: 37-43, 2007. 
13. Vakkila $\mathbf{J}$ and Lotze MT: Inflammation and necrosis promote tumour growth. Nat Rev Immunol 4: 641-648, 2004.

14. Lotze MT and Tracey KJ: High-mobility group box 1 protein (HMGB 1): nuclear weapon in the immune arsenal. Nat Rev Immunol 5: 331-342, 2005.

15. Taguchi A, Blood DC, del Toro G, et al: Blockade of RAGEamphoterin signalling suppresses tumour growth and metastases. Nature 405: 354-360, 2000.

16. Scaffidi P, Misteli T and Bianchi ME: Release of chromatin protein HMGB 1 by necrotic cells triggers inflammation. Nature 418: 191-195, 2002.

17. Dumitriu IE, Baruah P, Manfredi AA, Bianchi ME and RovereQuerini P: HMGB1: guiding immunity from within. Trends Immunol 26: 381-387, 2005.

18. Schlueter C, Weber H, Meyer B, et al: Angiogenetic signasling through hypoxia: HMGB1: an angiogenetic switch molecule. Am J Pathol 166: 1259-1263, 2005.

19. Choi YR, Kim H, Kang HJ, et al: Overexpression of high mobility group box 1 in gastrointestinal stromal tumours with KIT mutation. Cancer Res 63: 2188-2193, 2003.

20. Ishiguro H, Nakaigawa N, Miyoshi Y, Fujinami K, Kubota Y and Uemura H: Receptor for advanced glycation end products (RAGE) and its ligand, amphoterin are overexpressed and associated with prostate cancer development. Prostate 64: 92-100, 2005.

21. Guppy M: The hypoxic core: a possible answer to the cancer paradox. Biochem Biophys Res Commun 299: 676-680, 2002.

22. Gatenby RA and Gillies RJ: Why do cancers have high aerobic glycolysis? Nat Rev Cancer 4: 891-899, 2004.

23. Harris AL: Hypoxia - a key regulatory factor in tumour growth. Nat Rev Cancer 2: 38-47, 2002

24. Zhou J, Schmid T, Schnitzer S and Brune B: Tumour hypoxia and cancer progression. Cancer Lett 8: 10-21, 2006.

25. Tomes L, Emberley E, Niu Y, et al: Necrosis and hypoxia in invasive breast carcinoma. Breast Cancer Res Treat 81: 61-69, 2003.
26. Kim CH, Han SI, Lee SY, et al: Protein kinase C-ERK1/2 signal pathway switches glucose depletion-induced necrosis to apoptosis by regulating superoxide dismutases and suppressing reactive oxygen species production in A549 lung cancer cells. J Cell Physiol 211: 371-385, 2007.

27. Lim SC, Choi JE, Kim CH, et al: Ethyl pyruvate induces necrosis-to-apoptosis switch and inhibits high mobility group box protein 1 release in A549 lung adenocarcinoma cells. Int J Mol Med 20: 187-192, 2007.

28. Ahmad IM, Aykin-Burns N, Sim JE, et al: Mitochondrial $\mathrm{O}_{2}{ }^{*}-$ and $\mathrm{H}_{2} \mathrm{O}_{2}$ mediate glucose deprivation-induced stress in human cancer cells. J Biol Chem 280: 4254-4263, 2005.

29. Spitz DR, Sim JE, Ridnour LA, Galoforo SS and Lee YJ: Glucose deprivation-induced oxidative stress in human tumour cells. A fundamental defect in metabolism? Ann NY Acad Sci 899: 349-362, 2000.

30. Yung HW, Wyttenbach A and Tolkovsky AM: Aggravation of necrotic death of glucose-deprived cells by the MEK1 inhibitors U0126 and PD184161 through depletion of ATP. Biochem Pharmacol 15: 351-360, 2004

31. Sheu ML, Ho FM, Yang RS, et al: High glucose induces human endothelial cell apoptosis through a phosphoinositide 3-kinaseregulated cyclooxygenase- 2 pathway. Arterioscler Thromb Vasc Biol 25: 539-545, 2005.

32. Hamanaka Y, Mukai M, Shimamura M, et al: Suppression of $\mathrm{PI} 3 \mathrm{~K} / \mathrm{mTOR}$ pathway rescues LLC cells from cell death induced by hypoxia. Biochem Biophys Res Commun 330: 318-326, 2005.

33. Thyrell L, Hjortsberg L, Arulampalam V, et al: Interferon alpha-induced apoptosis in tumor cells is mediated through the phosphoinositide 3-kinase/mammalian target of rapamycin signaling pathway. J Biol Chem 279: 24152-24162, 2004. 